\title{
The Longitudinal Static Stability of an Aerodynamically Alleviated Marine Vehicle, a Mathematical Model
}

\author{
By Maurizio Collu, Minoo H. Patel and Florent Trarieux \\ Department of Offshore Engineering \& Renewable Energy, School of Engineering, \\ Cranfield University, Cranfield MK43 OAL, UK
}

An assessment of the relative speeds and payload capacities of airborne and waterborne vehicles highlights a gap which can be usefully filled by a new vehicle concept, utilizing both hydrodynamic and aerodynamic forces. A high speed marine vehicle equipped with aerodynamic surfaces is one such concept. In 1904, Bryan \& Williams published an article on the longitudinal dynamics of aerial gliders, and this approach remains the foundation of all the mathematical models studying the dynamics of airborne vehicles. In 1932, Perring \& Glauert presented a mathematical approach to study the dynamics of seaplanes experiencing the planing effect. From this work, planing theory has developed. The authors propose a unified mathematical model to study the longitudinal stability of a high speed planing marine vehicle with aerodynamic surfaces. A kinematics framework is developed. Then, taking into account the aerodynamic, hydrostatic and hydrodynamic forces, the full equations of motion, using a small perturbation assumption, are derived and solved specifically for this concept. This technique reveals a new static stability criterion that can be used to characterize the longitudinal stability of high speed planing vehicles with aerodynamic surfaces.

Keywords: marine vehicle; dynamics; stability; aerodynamic alleviation; wing in ground; planing

\section{Introduction}

Context During the last five decades, interest in High Speed Marine Vehicles (HSMV) has been increasing for both commercial and military use, leading to new configurations and further development of already existing configurations (Clark et al. 2004). To create vehicles capable of carrying more payload both farther and faster, many concepts have been proposed, and they can be classified analysing the force that can be employed to sustain the weight of a HSMV: hydrostatic lift (buoyancy), powered aerostatic lift, hydrodynamic lift.

Buoyancy is the lift force most commonly used by ships. Marine vehicles that exploit only buoyancy to sustain their weight are usually called displacement vessels. For high speed marine vehicles it is not feasible to use only buoyancy, as in this case the buoyancy force is proportional to the displaced water volume, and at high speed it is better to minimize this parameter, since as more vehicle volume (and the wetted surface) is immersed in the water the higher the hydrodynamic drag will be. 
The Air Cushion Vehicles (ACV) class use a cushion of air at a pressure higher than atmospheric to minimize contact with the water, thus minimizing hydrodynamic drag. The air cushion is not closed, and an air flux keeps the pressure in the cushion high. This system is called 'powered aerostatic lift'.

At high speeds a marine vehicle experiences 'hydrodynamic lift', due to the fact that the vehicle is planing over the water surface. This hydrodynamic lift supports the weight otherwise sustained by buoyancy or, through increasing the speed, can also replace in part or wholly the buoyancy force. Planing craft, high speed catamarans and other similar configurations use this principle to attain high speeds. If, instead of a simple planing hull, a surface similar to an aerofoil is used underwater, a hydrofoil is obtained. Basically, while in planing mode the hydrodynamic lift is generated by only one surface, the wetted surface of the hull, hydrofoils experience an effect similar to aerofoils, since the hydrodynamic lift is the difference between the pressure acting on the lower surface and the pressure on the upper surface.

A high speed marine vehicle can use two or all these three kinds of forces to sustain its weight. For example, a SES (Surface Effect Ship) consists of a catamaran hull configuration plus a powered air cushion with a front and a rear skirt in the space between the hulls. Therefore it experiences both hydrostatic and powered aerostatic lift.

There is another lift force that can be exploited to 'alleviate' the weight of the vehicle, leading to reduced buoyancy and therefore to decreased hydrodynamic drag: this is aerodynamic lift. There is an extreme case where the aerodynamic forces are sustaining $100 \%$ of the weight of the vehicle: WIG (Wing In Ground effect) vehicles.

A WIG vehicle is a vehicle designed to exploit the aerodynamic effect called "wing in ground effect". Extensive literature can be found on this effect and the vehicles exploiting it (Rozhdestvensky 2006), and only a brief introduction is given here. Given a conventional aerodynamic surface of fixed geometry, aerodynamic forces (lift, drag, and moment) acting on it depend on two variables: the speed of the surface relative to the air, and the angle of attack, defined as the angle between the chord of the wing and the speed direction. When this aerodynamic surface operates at a height above the surface, equal or lower than, roughly, one third of its span length, the aerodynamic forces experienced start to be dependent not only on the aforementioned parameters, but also on the height above the surface. The quality (plus or minus) and the quantity of these changes depend on the geometry of the wing, but in general it can be said that, reducing the height above the surface, with other parameters fixed, increases lift and decreases drag, leading to enhanced aerodynamic efficiency.

'Aerodynamically Alleviated Marine Vehicles' (AAMV)

An Aerodynamically Alleviated Marine Vehicle (AAMV) is a high speed marine vehicle designed to exploit, in its cruise phase, aerodynamic lift force, using one or more aerodynamic surfaces.

\section{Problem Statement}

This work illustrates a mathematical method to study the dynamics of an AAMV; a vehicle designed to exploit hydrodynamic and aerodynamic forces of the same order of magnitude, to sustain its weight. Methodologies for aircraft and marine craft exist and are well documented, but air and marine vehicles have always been investigated 
with a rather different approach. Marine vehicles have been studied analyzing very accurately hydrostatic and hydrodynamic forces, approximating very roughly the aerodynamic forces acting on the vehicle. On the contrary, the dynamics of Wing In Ground effect (WIG) vehicles has been modelled focusing mainly on aerodynamic forces, paying much less attention to hydrostatic and hydrodynamic forces.

An AAMV experiences aerodynamic and hydrodynamic forces of the same order of magnitude, therefore neither the high speed marine vehicles nor the airborne vehicles models of dynamics can cover and fully explain the AAMV dynamics. The main objective of this work is to bridge this gap by developing a new model of dynamics, in the small disturbances framework, consisting of a system of equations of motion that take into account the equal importance of aerodynamic and hydrodynamic forces. This mathematical model is further developed to estimate the static and dynamic stability of an AAMV.

\section{Literature review}

The hybrid nature of the model developed, hybrid between model of dynamics used for WIG vehicles and for planing craft, is mirrored by the literature review below. Furthermore, a section on literature review studying vehicles that can be classified as AAMV is presented.

Wing In Ground (WIG) vehicles Research on WIG vehicles has mainly been carried out in the former Soviet Union, where they were known as 'Ekranoplans'. The Central Hydrofoil Design Bureau, under the guidance of R. E. Alekseev, developed several test craft and the first production ekranoplans: Orlyonok and Lun types (Kolyzaev et al. 2000).

In the meantime, several research programs were undertaken in the west to better understand the peculiar dynamics of vehicles flying in ground effect (IGE). Irodov (1970) and Rozhdestvensky (1996) made important contributions to the development of WIG vehicles dynamic models.

In the 60's and the 70's Kumar (1968a, 1968b) started research in this area at Cranfield University. He carried out several experiments with a small test craft and provided the equations of motion, the dimensionless stability derivatives and studied the stability issues of a vehicle flying IGE.

Staufenbiel \& Bao-Tzang (1977) in the 70's carried out extensive work on the influence of aerodynamic surface characteristics on the longitudinal stability in wing in ground effect. Several considerations about the aerofoil shape, the wing planform and other aerodynamic elements were presented, in comparison with the experimental data obtained with the experimental WIG vehicle X-114 built by RheinFlugzeugbau in Germany in the 70's. The equations of motion for a vehicle flying IGE were defined, including non linear effects.

Hall, in 1994, extended the work of Kumar, modifying the equations of motion of the vehicle flying IGE, taking into account the influence of perturbations in pitch on the height above the surface.

More recently Chun \& Chang (2002) evaluated the stability derivatives for a 20 passenger WIG vehicle, based on wind tunnel results together with a vortex lattice method code. Using the work of Kumar and Staufenbiel, the static and dynamic stability characteristics were investigated. 
Planing craft Research on high speed planing started in the early twentieth century for the design of seaplanes (Perring \& Glauert 1932). Later research focused on applications to design planing boats and hydrofoil craft. During the period between 60's and 90's, many experiments were carried out and new theoretical formulations proposed.

Savitsky (1964) carried out an extensive experimental program on prismatic planing hulls and obtained some empirical equations to calculate forces and moments acting on planing vessels. He also provided simple computational procedures to calculate the running attitude of the planing craft (trim angle, draught), power requirements and also the stability characteristics of the vehicle.

Martin (1978) derived a set of equations of motion for the surge, pitch and heave degrees of freedom and demonstrated that surge can be decoupled from heave and pitch motion.

Troesch and Falzarano (Troesch 1992, Troesch \& Falzarano 1993) studied the nonlinear integro-differential equations of motion and carried out several experiments to develop a set of coupled ordinary differential equations with constant coefficients, suitable for modern methods of dynamical systems analysis.

Hicks et al. (1995) later extended their previous work and expanded the nonlinear hydrodynamic force equations of Zarnick (1978) using Taylor series up to the third order, obtaining a form of equation of motion suitable for path following or continuation methods.

Aerodynamically Alleviated Marine Vehicles (AAMV) In 1976, Shipps analysed a new kind of tunnel hull race boat. The advantages of this new configuration come from the aerodynamic lift. In 1978, Ward et al. published an article on the design and performance of a ram wing planing craft: the KUDU II. This vehicle, which consists in two planing sponsons separated by a wing section, was able to run at $78 \mathrm{kts}$ (almost $145 \mathrm{~km} / \mathrm{h}$ ), thanks to the aerodynamic lift alleviation. In 1978, Kallio performed comparative tests between the KUDU II and the KAAMA. The KAAMA is a conventional mono hull planing craft. The data obtained during comparative trials showed that the KUDU II pitch motion, in sea state 2, at about 40 to 60 knots, was about $30 \%$ to $60 \%$ lower than the conventional planing hull KAAMA.

In 1997, Doctors proposed a new configuration called 'Ekranocat' for which he mentioned the 'aerodynamic alleviation concept'. The weight of the catamaran was alleviated by aerodynamic lift, thanks to a more streamlined superstructure than in traditional catamarans. The theoretical analysis and computed results showed that a reduction in the total drag of around $50 \%$ can be obtained at very high speed, above 50 knots $(93 \mathrm{~km} / \mathrm{h})$.

In these references some experimental data, theoretical and computed results on vehicles which can be classified as AAMV are presented, but none of them present an analysis of the static stability of a vehicle having both hydrodynamic and aerodynamic surfaces. This is the gap the present work is aimed to bridge.

\section{The model}

In order to develop a mathematical model for the dynamics of an 'Aerodynamically Alleviated Marine Vehicle (AAMV)', a kinematics framework is proposed to describe the motion of the AAMV and the forces acting on it. Once a reference 


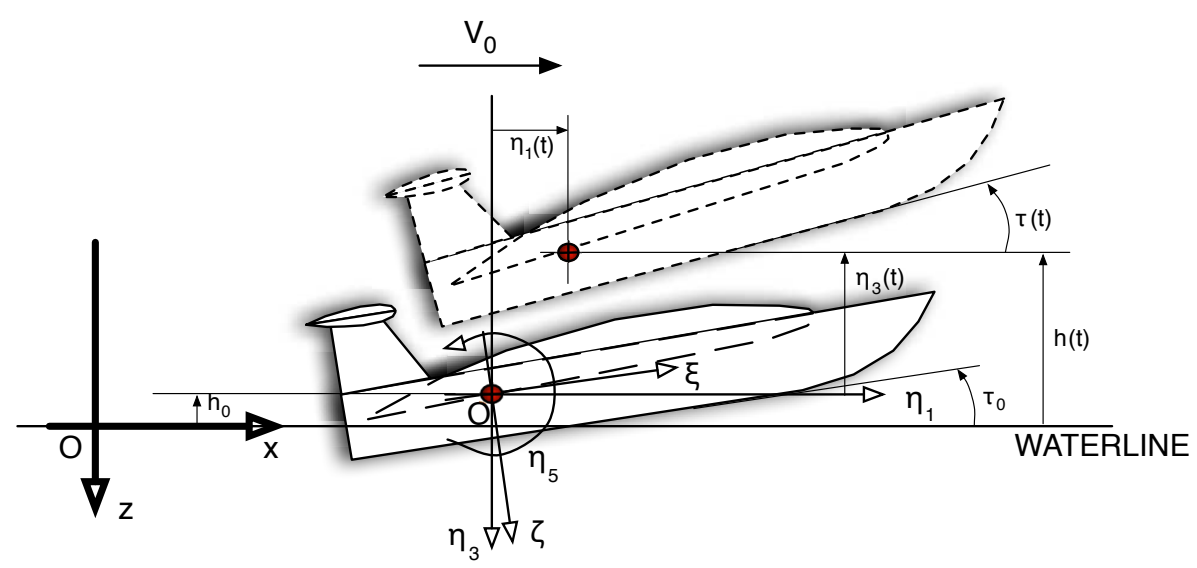

Figure 1. AAMV axis system

framework is established, it is necessary to narrow down possible configurations of the AAMV, since the qualitative and quantitative nature of the forces and moments acting on the AAMV depend on the elements that comprise its configuration.

\section{(a) Kinematics}

The study of kinematics requires a definition of coordinate frames, a notation to represent vehicle motion and a technique for a transformation between fixed and moving frames. To describe the motion of an AAMV and the forces acting on it, a number of different axis systems are used. Starting from the axis systems used for planing craft (Savitsky 1964, Martin 1978) and for WIG vehicles (Irodov 1970, Staufenbiel \& Bao-Tzang 1977), an earth-axis system and two body-axis system are presented below. They are all right-handed and orthogonal, as shown in figure 1. Dashed lines represent the vehicle in a disturbed state (rotation and displacements have been emphasized for clarity).

For body-axis systems, the origin $\mathrm{O}$ is taken to be coincident with the centre of gravity (CG) position of the AAMV in equilibrium state. The $x$ and $z$ axis lie in the longitudinal plane of symmetry, $x$ positive forward and $z$ positive downward. The direction of the $x$-axis depends on the body-axis system. Two are considered: aerohydrodynamic axes $\left(\eta_{1} \mathbf{O} \eta_{3}\right)$, the direction of the $x$-axis $\eta_{1}$ being parallel to the steady forward velocity $\mathrm{V}_{0}$, and geometric axes $(\xi \mathrm{O} \varsigma)$, the direction of the $x$-axis $\xi$ being parallel to a convenient geometric longitudinal datum (as the keel of the hull). Aero-hydrodynamic axes are used here as the counterpart of aerodynamic axes (called wind or wind-body axes in UK and stability axes in USA) used for airplanes. Usually the stability derivatives are calculated in this axis system.

The direction of the earth-axis systems $(x \mathrm{O} z)$ are fixed in space. The $z$-axis is directed vertically downward, the $x$-axis is directed forwards and parallel to the undisturbed waterline and the origin at the undisturbed waterline level. 


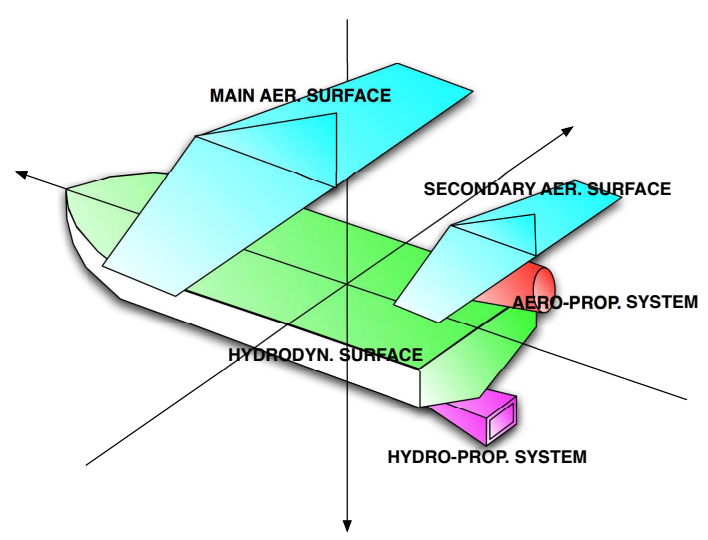

Figure 2. Class of configurations for the AAMV - Final choice

Table 1. AAMV configuration elements

\begin{tabular}{lll}
\hline Element & Number & Note \\
\hline Aerodynamic surface & 2 & with/out control surfaces \\
Hydrodynamic surface & 1 & $\begin{array}{l}\text { with/out control surfaces, } \\
\text { prismatic planing hull }\end{array}$ \\
Propulsion system & $1 /$ more & \begin{tabular}{l} 
aero- and/or hydro- propulsion system \\
\hline
\end{tabular}
\end{tabular}

\section{(b) Configuration}

The general approach of this work is to start from studies on WIG vehicles and high speed marine vehicles to derive an integrated system of equation of motion for an AAMV. This approach can be applied also to the choice of the AAMV configuration. A WIG vehicle's fundamental elements are the aerodynamic surfaces and the aero-propulsion system, while on a high speed marine vehicle the elements would be hydrostatic surfaces, hydrodynamic surfaces and a hydro-propulsion system.

During the course of the work, it was decided to limit the type of hydro-surfaces to only a prismatic planing hull (figure 2). Therefore the possible configurations was narrowed down, as shown in table 1.

The main difference is that, with the first class of configurations, hydrofoils could be represented. With the latter, only planing surfaces can be taken into account. Authors considered that the AAMV should also have the capability of free flight (or wing in ground flight), therefore the configuration with hydrofoils as hydrodynamic surfaces was not considered suitable.

Among all the other possible hydrostatic/hydrodynamic surfaces, a prismatic planing hull has been chosen, and the Savitsky planing hull model is used for this configuration (Savitsky 1964). The available literature on planing craft dynamics is extensive (Blake \& Wilson 2001) and the approaches used are somewhat similar to the approach used for WIG vehicles: this aspect makes the coupling of the airborne and waterborne dynamics simpler. Also if the majority of planing hulls used are nonprismatic, it has been demonstrated that the Savitsky approach is suitable also for non-prismatic hulls (Savitsky et al. 2007), and in particular in the preliminary phase of design. To estimate the equilibrium state, the starting point of the static and dynamic stability analysis presented in this work, the Savitsky method is chosen, 


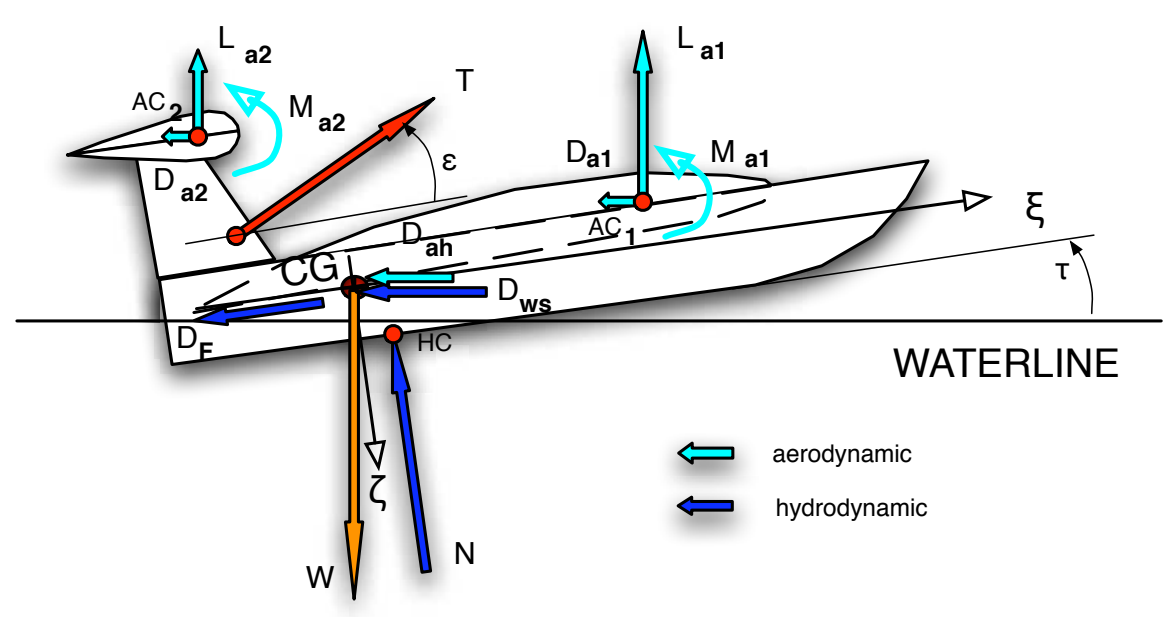

Figure 3. Forces and moments acting on a AAMV

Table 2. Forces and moment acting on an $A A M V$

\begin{tabular}{lll}
\hline Force & Symbol & Acting on \\
\hline Gravitational & $\mathrm{W}$ & Centre of gravity \\
Hydrostatic and hydrodynamic & $\mathrm{N}, \mathrm{D}_{w s}, \mathrm{D}_{F}$ & Hull \\
Aerodynamic & $\mathrm{L}_{a i}, \mathrm{D}_{a i}$, & aerodynamic surfaces \\
& $\mathrm{M}_{a i}, \mathrm{D}_{a h}$ & \\
Aerodynamic and hydrodynamic & & $\begin{array}{l}\text { aerodynamic surfaces } \\
\text { control systems }\end{array}$ \\
& & $\begin{array}{l}\text { and hull (control fixed analysis, } \\
\text { Aero- and/or hydro-propulsion }\end{array}$ \\
\hline
\end{tabular}

and along with it the prismatic planing monohull as the hydrostatic/hydrodynamic surface of the AAMV. The equilibrium state estimation method has been previously developed by the authors (Collu et al. 2008, Collu 2008).

\section{(c) Forces and Moments acting on the AAMV}

The forces and moments acting on the vehicle, after an external disturbance, are listed in table 2 and illustrated in figure 3.

Decoupling of Equations of Motion The AAMV, represented as a rigid body in space, and with control surfaces fixed, has 6 degrees of freedom. To describe its motion a set of six simultaneous differential equations of motion is needed. However, a decoupled system of equations of motion can be derived. For airplanes, in the frame of small perturbations approach, the lateral-longitudinal coupling is usually negligible. This is still valid for WIG vehicle (Chun \& Chang 2002). For planing craft, as demonstrated by Martin (1978), not only the lateral-longitudinal coupling is usually negligible, but also the surge motion can be decoupled from the heave and pitch motion. Therefore it is assumed that the AAMV has a negligible longitudinallateral coupling. In this work, the longitudinal motion of the AAMV is analysed, 
thus only the forces and moments acting in the longitudinal plane $x O z$ are taken into account: surge, heave forces and pitch moments. Following the nomenclature used for ships and airplanes, the force in the $\mathrm{x}$ direction is $X$, in the $\mathrm{z}$ direction is $Z$ and the moment about the y axis is $M$.

\section{(d) Forces and moments expressions}

The total force vector acting on the AAMV can be expressed as:

$$
\mathbf{F}=\mathbf{F}^{g}+\mathbf{F}^{a}+\mathbf{F}^{h}+\mathbf{F}^{c}+\mathbf{F}^{p}+\mathbf{F}^{d}
$$

where the components of each force are

$$
\mathbf{F}^{i}=\left[\begin{array}{lll}
X^{i} & Z^{i} & M^{i}
\end{array}\right]^{T}
$$

The total force is the sum of gravitational (g) force, aerodynamic (a) and hydrodynamic (h) forces, control (c) systems forces, propulsion (p) force and environment disturbances (d) forces.

When considering the motion of an airplane or a marine vehicle, after a small perturbation from a datum motion condition, it is usual to express aerodynamic and hydrodynamic forces and moments in Taylor expansions about their values at the datum motion state. The expansion can be nonlinear and expanded up to the n-th order, but in this work a linear expansion will be used. The linear approach has some limitations, but it is the result of a trade off. To study the dynamics of modern airborne vehicles, a non linear approach is used, but not in the early design phases (see, for example, Roskam 1989). In fact, in these phases a basic but solid understanding of the physics underpinning the static stability of a new concept vehicles is needed, and the linear approach is perfect for this task. The non linear approach would unnecessarily complicate the approach, hiding important aspects, and giving information too detailed at this stage. As regard hydrodynamic derivatives, linear methods are generally considered good enough to estimate the static stability boundaries (Troesch \& Falzarano 1993, Hicks et al. 1995).

As for airplanes and planing craft, forces and moments are assumed to depend on the values of the state variables and their derivatives with respect to time. Then, each force and moment is the sum of its value during the equilibrium state plus its expansion to take into account the variation after a small disturbance, which is:

$$
\begin{aligned}
& \mathbf{F}=\mathbf{F}_{0}+\mathbf{F}^{\prime} \\
& \mathbf{F}_{0}=\left[\begin{array}{lll}
X_{0} & Z_{0} & M_{0}
\end{array}\right]^{T} \\
& \mathbf{F}^{\prime}=\left[\begin{array}{lll}
X^{\prime} & Z^{\prime} & M^{\prime}
\end{array}\right]^{T}
\end{aligned}
$$

where the subscript $(0)$ denotes starting equilibrium state and superscript $\left({ }^{\prime}\right)$ denotes perturbation from the datum. Initially, the AAMV is assumed to maintain a Rectilinear Uniform Level Motion (RULM) with zero roll, pitch and yaw angles. In this particular motion, the steady forward velocity of the AAMV is $V_{0}$ and its components in the aero-hydrodynamic axis system are $\left[\dot{\eta}_{1,0}, \dot{\eta}_{3,0}\right]$, with $\dot{\eta}_{1,0}=V_{0}$ and $\dot{\eta}_{3,0}=0$, since this is a level motion (constant height above the surface).

Control, power and disturbances forces In this analysis, it is assumed that the controls are fixed (similar to the "fixed stick analysis" for airplanes). Then 
controls' forces and moments variations are equal to zero. The thrust is assumed not to vary during the small perturbation motion and it is equal to the total drag of the vehicle. The effects of environmental disturbances, like waves, are beyond the scope of this work; so a stable undisturbed environment is assumed.

$$
\left\{\begin{array}{l}
\mathbf{F}^{c}=\mathbf{F}_{0}^{c} \\
\mathbf{F}^{p}=\mathbf{F}_{0}^{p} \\
\mathbf{F}^{d}=\mathbf{0}
\end{array}\right.
$$

Gravitational force The gravitational contribution to the total force can be obtained by resolving the AAMV weight into the body axis system. Since the origin of the axis system is coincident with the CG of the AAMV, there is no weight moment about the y axis. Remembering that the equilibrium state pitch angle is equal to zero and the pitch angular perturbation $\theta^{\prime}$ is small, the gravitational contribution is

$$
\begin{aligned}
& \mathbf{F}^{g}=\mathbf{F}_{0}^{g}+\mathbf{F}^{g^{\prime}} \\
& \mathbf{F}_{0}^{g}=\left[\begin{array}{lll}
0 & m g & 0
\end{array}\right]^{T} \\
& \mathbf{F}^{g^{\prime}}=\left[\begin{array}{lll}
-m g \theta^{\prime} & 0 & 0
\end{array}\right]^{T}
\end{aligned}
$$

Aerodynamic forces Usually, to evaluate aerodynamic forces and moments, the state variables taken into account in their Taylor linear expansion are the velocity along the $\mathrm{x}$ and $\mathrm{z}$ axes $\left(\dot{\eta}_{1}\right.$ and $\left.\dot{\eta}_{3}\right)$ and the angular velocity about the y axis $\left(\dot{\eta}_{5}\right)$. Among the accelerations, only the vertical acceleration $\left(\ddot{\eta}_{3}\right)$ is taken into account in the linear expansion. Since the dynamics of a vehicle flying IGE depends also on the height above the surface, Kumar (1968, 1968b), Irodov (1970) and Staufenbiel \& Bao-Tzang (1977) introduced for WIG vehicles the derivatives with respect to height (h).

These derivatives can be evaluated knowing the geometrical and aerodynamics characteristics of the aerodynamic surfaces of the AAMV (Hall 1994). As shown by Chun and Chang (2002), the Taylor expansion to 1st order (linear model) is a good approach to have a first evaluation of the static and dynamic stability characteristics of the WIG vehicle.

The expansion of the generic aerodynamic force (moment) in the aero- hydrodynamic axis system $\left(\eta_{1} \mathrm{O} \eta_{3}\right)$ for a AAMV with a longitudinal plane of symmetry is

$$
\begin{aligned}
& \mathbf{F}^{a}=\mathbf{F}_{0}^{a}+\mathbf{F}^{a^{\prime}} \\
& \mathbf{F}_{0}^{a}=\left[\begin{array}{lll}
X_{0}^{a} & Z_{0}^{a} & M_{0}^{a}
\end{array}\right]^{T} \\
& \mathbf{F}^{a^{\prime}}=\quad\left[\begin{array}{c}
X_{h}^{a} \\
Z_{h}^{a} \\
M_{h}^{a}
\end{array}\right] \quad h^{\prime}+ \\
& +\left[\begin{array}{ccc}
X_{\dot{\eta}_{1}}^{a} & X_{\dot{\eta}_{3}}^{a} & X_{\dot{\eta}_{5}}^{a} \\
Z_{\dot{\eta}_{1}}^{a} & Z_{\dot{\eta}_{3}}^{a} & Z_{\dot{\eta}_{5}}^{a} \\
M_{\dot{\eta}_{1}}^{a} & M_{\dot{\eta}_{3}}^{a} & M_{\dot{\eta}_{5}}^{a}
\end{array}\right] \quad\left[\begin{array}{c}
\dot{\eta}_{1} \\
\dot{\eta}_{3} \\
\dot{\eta}_{5}
\end{array}\right]^{\prime}+ \\
& +\quad\left[\begin{array}{ccc}
0 & X_{\ddot{\eta}_{3}}^{a} & 0 \\
0 & Z_{\ddot{\eta}_{3}}^{a} & 0 \\
0 & M_{\ddot{\eta}_{3}}^{a} & 0
\end{array}\right] \quad\left[\begin{array}{c}
\ddot{\eta}_{1} \\
\ddot{\eta}_{3} \\
\ddot{\eta}_{5}
\end{array}\right]^{\prime}
\end{aligned}
$$


The superscript ${ }^{a}$ denotes "aerodynamic forces". $F_{j}$ denotes the derivative of the force (or moment) $F$ with respect to the state variable $j$, it corresponds to the partial differential $\partial F / \partial j$.

Hydrodynamic forces In Hicks et al. (1995), the nonlinear integro-differential expressions to calculate hydrodynamic forces and moments are expanded in a Taylor series, to third order. Therefore, equations of motion can be written as a set of ordinary differential equations with constant coefficients. The planing craft dynamics are highly non-linear, but the first step is to linearize the non-linear system of equations of motion and to calculate eigenvalues and eigenvectors, where variations are monitored with quasi-static changes of physical parameters, such as the position of the CG. This approach seems reasonable as a first step for the analysis of the AAMV dynamics too, for which a linear system of equations is developed.

The derivatives are usually divided into restoring coefficients (derivatives with respect to heave displacement and pitch rotation), damping coefficients (derivatives with respect to linear and angular velocities) and added mass coefficients (derivatives with respect to linear and angular accelerations). Martin (1978) and Troesch \& Falzarano (1993) showed that the added mass and damping coefficients are nonlinear functions of the motion but also that their nonlinearities are small compared to the restoring forces nonlinearities : therefore added mass and damping coefficients are assumed to be constant at a given equilibrium motion. Their value can be extrapolated from experimental results obtained by Troesch (1992). For the restoring coefficients, the linear approximation presented in Troesch and Falzarano (1993) will be followed:

$$
\mathbf{F}^{h, \text { restoring }}-\mathbf{F}_{0}^{h, \text { restoring }} \cong-[C] \underline{\eta}
$$

The coefficients of [C] can be determined using Savitsky's method for prismatic planing hull (Savitsky 1964) or the approach presented by Faltinsen (2005).

An approach to estimate added mass, damping and restoring coefficients is presented by Martin (1978). Furthermore, an alternative approach is to compute the added mass and damping coefficients as presented in Faltinsen (2005).

Then the expansion of the generic hydrodynamic force (moment) with respect to the aero-hydrodynamic axis system $\eta_{1} \mathrm{O} \eta_{3}$ is:

$$
\begin{aligned}
& \mathbf{F}^{h}=\mathbf{F}_{0}^{h}+\mathbf{F}^{h^{\prime}} \\
& \mathbf{F}_{0}^{h}=\left[\begin{array}{lll}
X_{0}^{h} & Z_{0}^{h} & M_{0}^{h}
\end{array}\right]^{T} \\
& \mathbf{F}^{h^{\prime}}=\left[\begin{array}{ccc}
0 & X_{\eta_{3}}^{h} & X_{\eta_{5}}^{h} \\
0 & Z_{\eta_{3}}^{h} & Z_{\eta_{5}}^{h} \\
0 & M_{\eta_{3}}^{h} & M_{\eta_{5}}^{h}
\end{array}\right] \quad\left[\begin{array}{c}
\eta_{1} \\
\eta_{3} \\
\eta_{5}
\end{array}\right]^{\prime}+ \\
& +\left[\begin{array}{ccc}
X_{\dot{\eta}_{1}}^{h} & X_{\dot{\eta}_{3}}^{h} & X_{\dot{\eta}_{5}}^{h} \\
Z_{\dot{\eta}_{1}}^{h} & Z_{\dot{\eta}_{3}}^{h} & Z_{\dot{\eta}_{5}}^{h} \\
M_{\dot{\eta}_{1}}^{h} & M_{\dot{\eta}_{3}}^{h} & M_{\dot{\eta}_{5}}^{h}
\end{array}\right] \quad\left[\begin{array}{c}
\dot{\eta}_{1} \\
\dot{\eta}_{3} \\
\dot{\eta}_{5}
\end{array}\right]^{\prime}+ \\
& +\left[\begin{array}{ccc}
X_{\ddot{\eta}_{1}}^{h} & X_{\ddot{\eta}_{3}}^{h} & X_{\ddot{\eta}_{5}}^{h} \\
Z_{\ddot{\eta}_{1}}^{h} & Z_{\ddot{\eta}_{3}}^{h} & Z_{\ddot{\eta}_{5}}^{h} \\
M_{\ddot{\eta}_{1}}^{h} & M_{\ddot{\eta}_{3}}^{h} & M_{\ddot{\eta}_{5}}^{h}
\end{array}\right] \quad\left[\begin{array}{c}
\ddot{\eta}_{1} \\
\ddot{\eta}_{3} \\
\ddot{\eta}_{5}
\end{array}\right]^{\prime}
\end{aligned}
$$

Article submitted to Royal Society 
The superscript ${ }^{h}$ denotes "hydrodynamic forces". $X_{\eta_{1}}, Z_{\eta_{1}}$ and $M_{\eta_{1}}$ are equal to zero since surge, heave and pitch moment are not dependent on the surge position of the AAMV.

Equilibrium state The equilibrium state has been already analysed (Collu et al. 2008, Collu 2008): here it is only briefly presented to make the necessary simplifications.

When an equilibrium state has been reached, by definition, all the accelerations are zero as well as all the perturbations velocities and the perturbation forces and moments. Then, using eq.s $4.3,4.4,4.5$ and 4.7 in eq. 4.2 :

$$
\left\{\begin{array}{l}
0=X_{0}^{a}+X_{0}^{h}+X_{0}^{c}+X_{0}^{p} \\
0=m g+Z_{0}^{a}+Z_{0}^{h}+Z_{0}^{c}+Z_{0}^{p} \\
0=M_{0}^{a}+M_{0}^{h}+M_{0}^{c}+M_{0}^{p}
\end{array}\right.
$$

\section{(e) System of Equations of Motion}

The generalized system of equations of motion (in 6 degrees of freedom) of a rigid body with a left/right (port/starboard) symmetry is linearized in the frame of small-disturbance stability theory. The starting equilibrium state is a RULM, with a steady forward velocity equal to $V_{0}$. The total velocity components of the AAMV in the disturbed motion are (evaluated in the Earth-axis system):

$$
\left[\begin{array}{c}
\dot{\eta}_{1} \\
\dot{\eta}_{2} \\
\dot{\eta}_{3} \\
\dot{\eta}_{4} \\
\dot{\eta}_{5} \\
\dot{\eta}_{6}
\end{array}\right]=\left[\begin{array}{c}
V_{0}+\dot{\eta}_{1}^{\prime} \\
\dot{\eta}_{2}^{\prime} \\
\dot{\eta}_{3}^{\prime} \\
\dot{\eta}_{4}^{\prime} \\
\dot{\eta}_{5}^{\prime} \\
\dot{\eta}_{6}^{\prime}
\end{array}\right]
$$

By definition for small disturbances, all the linear and the angular disturbance velocities (denoted with ') are small quantities: therefore, substituting eq. 4.9 in the generalized 6 degrees of freedom equations of motion, and eliminating the negligible terms, the linearized equations of motion can be expressed as

$$
\left\{\begin{aligned}
m \ddot{\eta}_{1}^{\prime} & =X \\
m\left(\ddot{\eta}_{2}^{\prime}+\dot{\eta}_{6}^{\prime} V_{0}\right) & =Y \\
m\left(\ddot{\eta}_{3}^{\prime}-\dot{\eta}_{5}^{\prime} V_{0}\right) & =Z \\
I_{44} \ddot{\eta}_{4}^{\prime}-I_{46} \ddot{\eta}_{6}^{\prime} & =L \\
I_{55} \ddot{\eta}_{5}^{\prime} & =M \\
I_{66} \ddot{\eta}_{6}^{\prime}-I_{64} \ddot{\eta}_{4}^{\prime} & =N
\end{aligned}\right.
$$

If the system of equations is decoupled, the longitudinal (symmetric) linearized equations of motion are

$$
\left\{\begin{aligned}
m \ddot{\eta}_{1}^{\prime} & =X \\
m\left(\ddot{\eta}_{3}^{\prime}-\dot{\eta}_{5}^{\prime} V_{0}\right) & =Z \\
I_{55} \ddot{\eta}_{5}^{\prime} & =M
\end{aligned}\right.
$$




\section{(f) Longitudinal Linearized System of Equations of Motion}

Taking into account eq. 4.8, the longitudinal linearized equations of motion (eq. 4.11) written in the aero-hydrodynamic axis system can be rearranged as:

$$
[A] \underline{\ddot{\eta}}+[B] \underline{\dot{\eta}}+[C] \underline{\eta}+[D] h=\mathbf{0}
$$

where

$$
\underline{\eta}=\left[\begin{array}{l}
\eta_{1} \\
\eta_{3} \\
\eta_{5}
\end{array}\right]
$$

and $h$ is the (perturbated) height above the waterline.

The matrix $[\mathrm{A}]$ is the sum of the mass matrix, the hydrodynamic added mass derivatives and the aerodynamic "added mass" terms (usually in aerodynamics they are referred to as simply "acceleration derivatives"), whereas in the hydrodynamic case these terms are much more significant.

$$
[A]=\left[\begin{array}{ccc}
m-X_{\ddot{\eta}_{1}}^{h} & -X_{\ddot{\eta}_{3}}^{a}-X_{\ddot{\eta}_{3}}^{h} & -X_{\ddot{\eta}_{5}}^{h} \\
-Z_{\ddot{\eta}_{1}}^{h} & m-Z_{\ddot{\eta}_{3}}^{a}-Z_{\ddot{\eta}_{3}}^{h} & -Z_{\ddot{\eta}_{5}}^{h} \\
-M_{\ddot{\eta}_{1}}^{h} & -M_{\ddot{\eta}_{3}}^{a}-M_{\ddot{\eta}_{3}}^{h} & I_{55}-M_{\ddot{\eta}_{5}}^{h}
\end{array}\right]
$$

$[\mathrm{B}]$ is the damping matrix and is defined as:

$$
[B]=\left[\begin{array}{ccc}
-X_{\dot{\eta}_{1}}^{a}-X_{\dot{\eta}_{1}}^{h} & -X_{\dot{\eta}_{3}}^{a}-X_{\dot{\eta}_{3}}^{h} & -X_{\dot{\eta}_{5}}^{a}-X_{\dot{\eta}_{5}}^{h} \\
-Z_{\dot{\eta}_{1}}^{a}-Z_{\dot{\eta}_{1}}^{h} & -Z_{\dot{\eta}_{3}}^{a}-Z_{\dot{\eta}_{3}}^{h} & -Z_{\dot{\eta}_{5}}^{a}-Z_{\dot{\eta}_{5}}^{h} \\
-M_{\dot{\eta}_{1}}^{a}-M_{\dot{\eta}_{1}}^{h} & -M_{\dot{\eta}_{3}}^{a}-M_{\dot{\eta}_{3}}^{h} & -M_{\dot{\eta}_{5}}^{a}-M_{\dot{\eta}_{5}}^{h}
\end{array}\right]
$$

$[\mathrm{C}]$ is the restoring matrix and is defined as:

$$
[C]=\left[\begin{array}{ccc}
0 & -X_{\eta_{3}}^{h} & -m g-X_{\eta_{5}}^{h} \\
0 & -Z_{\eta_{3}}^{h} & -Z_{\eta_{5}}^{h} \\
0 & -M_{\eta_{3}}^{h} & -M_{\eta_{5}}^{h}
\end{array}\right]
$$

The matrix $[\mathrm{D}]$ represents the wing in ground effect, to take into account the influence of the height above the surface on the aerodynamic forces.

$$
[D]=\left[\begin{array}{c}
-X_{h}^{a} \\
-Z_{h}^{a} \\
-M_{h}^{a}
\end{array}\right]
$$

(g) Cauchy standard form of the Equations of Motion

By defining a state space vector $\underline{\nu}$ as

$$
\underline{\nu}=\left[\begin{array}{llllll}
\dot{\eta_{1}} & \dot{\eta_{3}} & \dot{\eta_{5}} & \eta_{3} & \eta_{5} & \eta_{0}
\end{array}\right]^{T}
$$

the system of equations eq. 4.12 can be transformed to the Cauchy standard form (or state-space form). The state space vector has six variables while the system of equations eq. 4.12 has only 3 equations. The remaining 4 equations are:

$$
\begin{cases}\frac{\partial\left(\eta_{3}\right)}{\partial t} & =\dot{\eta}_{3} \\ \frac{\partial\left(\eta_{5}\right)}{\partial t} & =\dot{\eta}_{5} \\ \frac{\partial(h)}{\partial t}=\frac{\partial\left(\eta_{0}\right)}{\partial t} & =-\dot{\eta}_{3}+V_{0} \eta_{5}\end{cases}
$$

Article submitted to Royal Society 
Therefore the system is:

$$
\left[A_{S S}\right] \underline{\dot{\nu}}=\left[B_{S S}\right] \underline{\nu}
$$

where

$$
\left[A_{S S}\right]=\left[\begin{array}{cccc}
{[A]} & \multicolumn{3}{c}{[0]_{3 \times 3}} \\
& 1 & 0 & 0 \\
{[0]_{3 \times 3}} & 0 & 1 & 0 \\
& 0 & 0 & 1
\end{array}\right]
$$

and

$$
\left[B_{S S}\right]=\left[\begin{array}{ccccccc} 
& \multicolumn{1}{c}{0} & -m g & \\
& -[B] & & -C_{33} & -C_{35} & -[D] \\
& \multicolumn{1}{c}{-C_{53}} & -C_{55} & \\
0 & 1 & 0 & 0 & 0 & 0 \\
0 & 0 & 1 & 0 & 0 & 0 \\
0 & -1 & 0 & 0 & V_{0} & 0
\end{array}\right]
$$

The system of equations of motion in state-space form is:

$$
\underline{\dot{\nu}}=[H] \underline{\nu}
$$

where

$$
[H]=\left[A_{S S}\right]^{-1} \quad\left[B_{S S}\right]
$$

Now it is possible to analyse the static and dynamic stability of a AAMV configuration and the influence of the configuration characteristics on the AAMV dynamics.

\section{Static stability}

Analyzing the forces and moments under the small disturbances hypothesis, the static stability of an AAMV is derived using the Routh-Hurwitz criterion.

In particular, Staufenbiel \& Bao-Tzang (1977) showed how the last coefficient $A_{0}$ of the characteristic polynomial of a WIG vehicle can be used to estimate its static stability. In general, given the characteristic polynomial of a system:

$$
A_{n} s^{n}+A_{n-1} s^{n-1}+\ldots+A_{1} s^{1}+A_{0}=0
$$

if the condition

$$
\frac{A_{0}}{A_{n}}>0
$$

with $A_{n}>0$, is fulfilled, the system is statically stable.

(a) AAMV characteristic polynomial and static stability condition

In $\S 4$ the mathematical model is developed to study the longitudinal dynamics of an AAMV. A system of ordinary differential equations of motion is derived for the longitudinal plane in the frame of small-disturbance stability theory. Starting from this model, the Routh-Hurwitz condition is used to derive a mathematical expression to estimate the AAMV static stability. 
Complete order system By defining a state space vector $\underline{\nu}$ as

$$
\underline{\nu}=\left[\begin{array}{llllll}
\dot{\eta_{1}} & \dot{\eta_{3}} & \dot{\eta_{5}} & \eta_{3} & \eta_{5} & \eta_{0}
\end{array}\right]^{T}
$$

the system of equations of motion can be rearranged in the Cauchy standard form (or state-space form), showed in eq. 4.19. The characteristic polynomial of the complete order system can be derived:

$$
A_{6} s^{6}+A_{5} s^{5}+A_{4} s^{4}+A_{3} s^{3}+A_{2} s^{2}+A_{1} s^{1}+A_{0}=0
$$

With $A_{6}=1$, the static stability is assured when:

$$
A_{0}=\frac{\text { num }_{0}}{\Delta}>0
$$

where num $_{0}$ is equal to

$$
\text { num }_{0}=V_{0}\left[D_{10}\left(B_{31} C_{53}-B_{51} C_{33}\right)-B_{11}\left(C_{35} D_{50}-C_{53} D_{30}\right)\right]
$$

and $\Delta$ is equal to

$$
\begin{aligned}
& \Delta=\left(I_{55}+A_{55}\right)\left[m^{2}+m\left(A_{11}+A_{33}\right)+A_{11} A_{33}-A_{31} A_{13}\right]+ \\
& -\left(m+A_{11}\right) A_{53} A_{35}-\left(m+A_{33}\right) A_{51} A_{15}+A_{53} A_{31} A_{15}+A_{51} A_{13} A_{35}
\end{aligned}
$$

$A_{i j}, B_{i j}, C_{i j}$, and $D_{i j}$ stability derivatives are illustrated, respectively, in eq. 4.13, 4.14, 4.15, and 4.16.

Reduced order system This mathematical method has to be validated against experimental data. Unfortunately, no experimental data on static stability of a AAMV configuration is available in the public domain.

To plan experiments to obtain these data, it is necessary to have a physical insight of the condition stated in eq. 5.2. This condition, applied to the complete order system in eq. 5.5, is relatively complex. Assuming that the surge degree of freedom $\left(\eta_{1}\right)$ can be decoupled from heave $\left(\eta_{3}\right)$ and $\left(\eta_{5}\right)$ pitch degrees of freedom, a simplified version of the condition in eq. 5.5 can be obtained, leading to a better physical insight.

The mathematical model of the dynamics here developed starts from the systems of equations of motion for WIG vehicles and planing craft. As regards the dynamics of a planing craft, Martin (1978) demonstrated that the surge motion can be decoupled from the heave and pitch motion. For the dynamics of WIG vehicles, Rozhdestvensky (1996) proposed a reduced order system where the surge motion is decoupled from heave and pitch motion.

By defining the reduced order state space vector $\underline{\nu}$ as

$$
\underline{\nu}=\left[\begin{array}{lllll}
\dot{\eta}_{3} & \dot{\eta}_{5} & \eta_{3} & \eta_{5} & \eta_{0}
\end{array}\right]^{T}
$$

the Cauchy standard form (or state-space form) of the reduced order system is obtained. The characteristic polynomial can be derived:

$$
A_{5} s^{5}+A_{4} s^{4}+A_{3} s^{3}+A_{2} s^{2}+A_{1} s^{1}+A_{0}=0
$$

With $A_{5}=1$, the static stability is assured when

$$
A_{0}=\frac{V_{0}\left(C_{33} D_{50}-C_{53} D_{30}\right)}{\left(A_{55}+I_{55}\right)\left(A_{3}+m\right)-A_{53} A_{35}}>0
$$




\section{(b) Reduced order static stability: physical insight}

Each coefficient in eq. 5.10 is the derivative with respect to: accelerations $\left(\mathrm{A}_{i j}\right)$, heave position $\left(\mathrm{C}_{i j}\right)$, and height above the surface $\left(\mathrm{D}_{i j}\right)$ of the sum of aerodynamic and hydrodynamic forces (and moments). Referring to $\S 4.11$, remembering that the superscript ' $a$ ' stands for aerodynamic and ' $h$ ' for hydrodynamic, and that $\mathrm{Z}$ is the heave force (positive downward) and $M$ the pitch moment (positive bow up), the coefficients are equal to:

$$
\begin{aligned}
& A_{33}=A_{33}^{a}+A_{33}^{h}=-Z_{\ddot{\eta} 3}^{a}-Z_{\ddot{\eta} 3}^{h} \\
& A_{35}=A_{35}^{a}+A_{35}^{h}=-Z_{\ddot{\eta} 5}^{a}-Z_{\ddot{\eta} 5}^{h} \\
& A_{53}=A_{53}^{a}+A_{53}^{h}=-M_{\ddot{\eta} 3}^{a}-M_{\ddot{\eta} 3}^{h} \\
& A_{55}=A_{55}^{a}+A_{55}^{h}=-M_{\ddot{\eta} 5}^{a}-M_{\ddot{\eta} 5}^{h} \\
& C_{33}=C_{33}^{h}=-Z_{\eta 3}^{h} \quad, \quad C_{33}^{a}=0 \\
& C_{53}=C_{53}^{h}=-M_{\eta 3}^{h} \quad, \quad C_{53}^{a}=0 \\
& D_{30}=D_{30}^{a}=-Z_{\eta 0}^{a} \quad, \quad D_{30}^{h}=0 \\
& D_{50}=D_{53}^{a}=-M_{\eta 0}^{a} \quad, \quad D_{50}^{h}=0
\end{aligned}
$$

The aerodynamic derivatives can be estimated with the approach presented in Hall (1994) and the hydrodynamic derivatives with expressions presented by Martin (1978) and by Faltinsen (2005). Using these expressions for the configuration presented in $\S 4 \mathrm{~b}$ we have

$$
\left(A_{55}+I_{55}\right)\left(m+A_{33}\right)-A_{53} A_{35}>0
$$

therefore, since the denominator of eq. 5.10 is greater than zero, the static stability condition of the reduced order becomes

$$
\frac{D_{50}}{D_{30}}-\frac{C_{53}}{C_{33}}>0
$$

Similarity with WIG vehicles To better understand the condition expressed in eq. 5.14, a parallel with WIG vehicles static stability criteria is illustrated. The static stability condition derived by Staufenbiel \& Bao-Tzang (1977) and Irodov (1970) is:

$$
\frac{M_{w}}{Z_{w}}-\frac{M_{h}}{Z_{h}}<0
$$

that, using the present nomenclature corresponds to the condition

$$
\frac{B_{53}}{B_{33}}-\frac{D_{50}}{D_{30}}<0
$$

$\mathrm{M}_{w}$ and $\mathrm{M}_{h}$ are the derivatives of pitch moment with respect to the heave velocity and the height above the surface, $\mathrm{Z}_{w}$ and $\mathrm{Z}_{h}$ are the heave force same derivatives. Staufenbiel \& Bao-Tzang and Irodov define $\mathrm{M}_{w} / \mathrm{Z}_{w}$ also as the aerodynamic centre of pitch and $\mathrm{M}_{h} / \mathrm{Z}_{h}$ as the aerodynamic centre in height. Remembering that positive abscissa means ahead of the CG, the condition in eq. 5.15 and 5.16 can be expressed as (Rozhdestvensky 2006): 


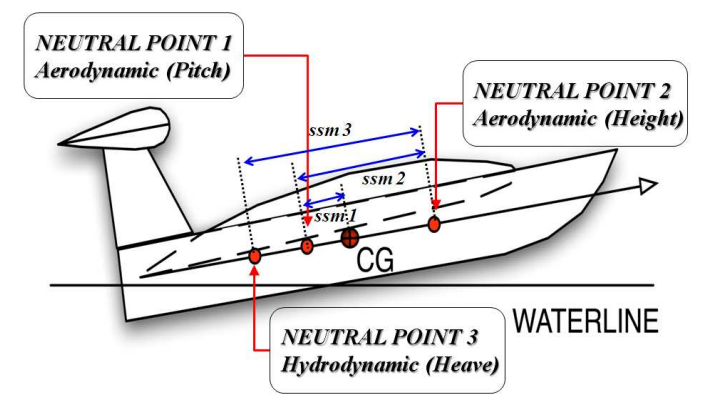

Figure 4. AAMV static stability: graphical analysis

"the (aerodynamic) centre in height should be located upstream of the (aerodynamic) centre in pitch."

Dividing the lift due to a variation of the pitch angle $\left(\Delta L_{\text {alpha }}\right)$ from the lift due to a variation of the height above the surface $\left(\Delta L_{\text {height }}\right)$, condition in eq. 5.15 states that the point of action of force $\Delta L_{\text {height }}$ should be located upstream the point of action of force $\Delta L_{\text {alpha }}$.

AAMV Static stability criterion (reduced order) As regard the AAMV, using expressions 5.12, the static stability condition in eq. 5.14 can be expressed as:

$$
\frac{M_{\eta 0}^{a}}{Z_{\eta 0}^{a}}-\frac{M_{\eta 3}^{h}}{Z_{\eta 3}^{h}}>0
$$

The first term $M_{\eta 0}^{a} / Z_{\eta 0}^{a}$ is the analogue of the aerodynamic centre in height of WIG vehicles. Author propose for the second term the name 'hydrodynamic centre in heave', so that eq. 5.17 can be also expressed as:

"the hydrodynamic centre in heave should be located downstream of the aerodynamic centre in height."

As before, dividing the hydrodynamic lift due to a heave variation $\left(\Delta L_{h y d}\right)$ from the lift due to a variation of the height above the surface $\left(\Delta L_{\text {height }}\right)$, it needs to be checked that the point of action of $\Delta L_{h y d}$ is located upstream the point of action of $\Delta L_{h y d}$.

(c) Reduced order system static stability: graphical insight

To better understand conditions expressed in eq. 5.17, a graphical representation of the aerodynamic centre in height, the aerodynamic centre in pitch and the hydrodynamic centre in heave is given in figure 4 .

For a conventional aircraft the longitudinal static stability, without considering the influence causing a change in the aerodynamic control surfaces (the so called 'fixed stick analysis'), is determined only by the position of the 'neutral point 1', called the 'aerodynamic centre in pitch'. This point can be imagined as the point where the aerodynamic forces, due to an external disturbance of the pitch angle, act. To have an airplane statically stable, the aerodynamic centre in pitch should be located rearward with respect to the CG, and the distance between the neutral point 1 and the CG is called static stability margin $\left(s s m_{1}\right)$, since it is a measure of how statically stable the airplane is. 
For wing in ground effect vehicles there is an additional neutral point, designated 'neutral point 2', due to the fact that aerodynamic forces depend also on the height of the vehicle above the surface. As stated by Staufenbiel \& Bao-Tzang, Irodov and others, to have a statically stable WIG vehicle, it should fulfill the condition 'neutral point 1 rearward of the CG' plus another condition: the neutral point 2 should be located upstream with respect to the neutral point 1 . The distance between the two neutral points can be assumed as a second static stability margin, indicated as $\mathrm{ssm}_{2}$.

For an aerodynamically alleviated marine vehicle a third neutral point exists, due to the fact that the forces depend on: (a) the pitch angle as for airplane and WIG vehicles, (b) the height above the surface as in WIG vehicles, and (c) on the heave position. In fact hydrodynamic forces depend heavily on the heave position of the AAMV. Furthermore, the condition expressed in eq. 5.17 is represented here by the fact that 'neutral point 3', also called 'hydrodynamic centre in heave', is located downstream with respect to the 'neutral point 2', also called aerodynamic centre in height. As for previous cases, the distance between the neutral point 2 and the neutral point 3 can be assumed as a measure of the static stability margin in heave $\left(s s m_{3}\right)$. The condition for the AAMV static stability is derived using the reduced order system of equations of motion, following an approach already used for WIG vehicles. It should be noted that it is useful for the preliminary design phase of the vehicle and to have a quick physical insight of the static stability of a AAMV.

\section{Conclusions}

In the last few decades, interest in high speed marine vehicles (HSMV) has been increasing, leading to several new configuration concepts. Among these, the 'aerodynamic alleviation concept' (Doctors 1997) consists of using one or more aerodynamic surfaces to alleviate the weight of the marine vehicle. Basically, the advantages are: (a) a total drag, at high speed, up to 20-30\% lower than the same marine vehicle without aerodynamic surfaces (Collu 2008), (b) vertical and angular pitch accelerations, at high speed, 30-60\% lower than conventional HSMV (Kallio 1978), and (c) a vehicle bridging the speed gap and payload gap between conventional high speed marine vehicles and airplanes.

To classify this configuration concept, the new abbreviation AAMV, 'Aerodynamically Alleviated Marine Vehicle' is used. Being a relatively recent configuration concept, it lacks a specifically developed mathematical framework to study its dynamics. The AAMV experiences aerodynamic and hydrodynamic forces of the same order of magnitude, therefore the mathematical frameworks developed separately so far for high speed marine vehicles and airplanes are not suitable. This paper presents an integrated mathematical framework specifically for an AAMV configuration.

AAMV System of Equations of Motion A mathematical model of the longitudinal dynamics of a AAMV configuration, developed in the small disturbances framework, is presented. Coupling the systems of equations of motion, available in literature, used for Wing in Ground effect (WIG) vehicles and for high speed planing craft, authors derived a new system of equations of motion. This mathematical model takes into account aerodynamic, hydrodynamic and hydrostatic stability derivatives, leading to a new dynamics. In fact, also if this AAMV mathematical model is obtained by combining the WIG vehicles and the planing craft dynamics, 


\begin{tabular}{ccc}
\hline $\begin{array}{c}\text { Vehicle } \\
\text { configuration }\end{array}$ & $\begin{array}{c}\text { System of } \\
\text { Equations of Motion }\end{array}$ & Roots \\
\hline Airplane & $\begin{array}{c}4 \text { equations } \\
\frac{\partial x}{\partial t}, \frac{\partial z}{\partial t}, \frac{\partial \theta}{\partial t}, \theta\end{array}$ & $\begin{array}{c}\text { oscillatory sol.s: } \\
\text { phugoid, SPPO }\end{array}$ \\
Planing craft & 4 equations & 2 oscillatory sol.s: \\
& $\frac{\partial z}{\partial t}, z, \frac{\partial \theta}{\partial t}, \theta$ & porposing (least stable root) \\
WIG vehicles & 5 equations & 2 oscillatory sol.s, 1 real root: \\
& $\frac{\partial x}{\partial t}, \frac{\partial z}{\partial t}, \frac{\partial \theta}{\partial t}, \theta, h$ & phugoid, SPPO, subsidence mode \\
AAMV & 6 equations & (for the reduced order system, \\
& $\frac{\partial x}{\partial t}, \frac{\partial z}{\partial t}, z$, & without $\frac{\partial x}{\partial t}$, \\
$\frac{\partial y}{\partial t}, \theta, h$ & 2 oscillatory sol.s, 1 real root) \\
\hline
\end{tabular}

Table 3. Comparison between the dynamics characteristics of conventional configurations and the $A A M V$ dynamics

the resultant dynamics is not simply the sum of these dynamics. As shown in table 3 , the system of equations of motion developed by authors proposes a new dynamic feature, with a potential new mode of oscillation and a more complex dynamics with respect to WIG vehicles, planing craft and conventional airplanes.

AAMV static stability criterion The AAMV dynamics differ substantially from planing craft dynamics and from airplanes and WIG vehicles dynamics, and the static stability of a AAMV configuration is analysed, and a new static stability criterion is proposed.

Briefly, in the longitudinal plane, airplanes possess one neutral point, called aerodynamic neutral point in pitch (or also neutral point): if this point is rearward with respect to the CG of the vehicle, the airplane is statically stable. By contrast, WIG vehicles have an additional neutral point, the aerodynamic neutral point in height, due to the fact that aerodynamic forces depend also on the vehicle's height above the surface. To have a WIG vehicle statically stable in height, this second neutral point should be upstream with respect to the first neutral point, as investigated by Staufenbiel \& Bao-Tzang (1977), Irodov (1970), and Rozhdestvensky (1996). For a aerodynamically alleviated marine vehicles (AAMV), a third neutral point exists, due to the fact that the forces depend on: the pitch angle, as for an airplane and WIG vehicles, the height above the surface, as in WIG vehicles, and the heave position. In fact the hydrodynamic forces depend heavily on the heave position of the AAMV. The condition expressed by eq. 5.17 can be expressed saying that the third neutral point, also called the hydrodynamic centre in heave, should be located downstream with respect to the neutral point 2, to have an AAMV that is statically stable in heave. The relative position of each point is shown in figure 4 . In airplane dynamics, the distance between the CG and the (aerodynamic) neutral point (in pitch) is called 'static stability margin' $\left(s s m_{1}\right)$. Following the same approach, the distance between the second neutral point and the first neutral point can be called 'static stability margin in height' $\left(s s m_{2}\right)$. As already said, for a AAMV configuration a third neutral point exists, and the distance between the second and the third neutral point can be called 'hydrodynamic static stability margin in heave' $\left(\mathrm{ssm}_{3}\right)$. 


\section{References}

Blake, J. I. R. \& Wilson, P. A. 2001 A visual experimental technique for planing craft performance. Trans. R. Inst. Naval Architects 143, 393-404.

Bryan, G. H. \& Williams, W. E. 1904 The longitudinal stability of aerial gliders. Proc. R. Soc. Lond. 73, 100-116.

Clark, D. J., Ellsworth, W. M. \& Meyer, J. R. 2004 The quest for speed at sea. Technical Digest, April 2004, Naval Surface Warfare Center, Carderock Division.

Chun, H. H. \& Chang, C. H. 2002 Longitudinal stability and dynamic motion of a small passenger WIG craft. Ocean Eng. 29, 1145-1162.

Collu, M., Patel, M. H. \& Trarieux, F. 2008 A mathematical model to analyse the static stability of hybrid (aero-hydrodynamically supported) vehicles. In 8 th Symposium on High Speed Marine Vehicles, Naples, Italy, 21 May 2008, pp. 148-161.

Collu, M. 2008 Marine vehicles with aerodynamic surfaces: dynamics mathematical model development. Ph.D. thesis, Cranfield University, Cranfield.

Doctors, L. J. 1997 Analysis of the efficiency of an ekranocat: a very high speed catamaran with aerodynamic alleviation. In Int. Conf. on Wing in Ground Effect Craft, London, UK, 4 December 1997.

Faltinsen, O. M. 2005 Hydrodynamics of high - speed vehicles, pp. 454. Cambridge University Press.

Hall, I. A. 1994 An investigation into the flight dynamics of wing in ground effect aircraft operating in aerodynamic flight. M.Sc. thesis, Cranfield University, Cranfield.

Hicks, J. D., Troesch, A. W. \& Jiang, C. 1995 Simulation and Nonlinear Dynamics Analysis of Planing Hulls. J. Offshore Mech. Arct. Eng. 117, 38-45. (doi:10.1115/1.2826989)

Irodov, R. D. 1970 Kriterii prodol'noy ustoychivosti ekranoplana. TsAGI im. N. Ye. Zhukovskij 1 , 4 63-72. (Transl. WIG longitudinal stability criteria, Central Institute of Aerohydrodynamics )

Kallio, J. A. 1978 Results of Full Scale Trials on Two High Speed Planing Craft (Kudu II and Kaama). Report no. DTNSRDC/SPD-0847-01, David W. Taylor Naval Ship Research and Development Center, Carderock, Md.

Katayama, T. \& Ikeda, Y. 1999 Hydrodynamic Forces Acting on Porpoising Craft at High-Speed. J. Ship Ocean Technol. 3, 17-26.

Kolyzaev, B., Zhukov, V., Maskalik, A. 2000 Ekranoplans, peculiarity of the theory and design. Saint Petersburg: Saint Petersburg Sudostroyeniye.

Kumar, P. 1968a Stability of ground effect vehicles. Report No. Aero 198, Cranfield College of Aeronautics.

Kumar, P. $1968 b$ On the longitudinal dynamic stability of a ground effect wing, Report No. Aero 202, Cranfield College of Aeronautics.

Martin, M. 1978 Theoretical Determination of Porpoising Instability of High-Speed Planing Boats. J. Ship Res. 22, 32-53

Moore, N., Wilson, P. A. \& Peters, A. J. 2002 An investigation into wing in ground effect airfoil geometry. In Symposium on Challenges in Dynamics, System Identification, Control and Handling Qualities for Land, Air, Sea and Space Vehicles, Berlin, Germany, 13 May 2002, pp.11.1-11.20.

Payne, P. R. 1974 Coupled Pitch and Heave Porpoising Instability in Hydrodynamic Planing. J. Hydronautics 8, 58-71. (doi: 10.2514/3.62979)

Perring, W. G. A. \& Glauert, H. 1932 Stability on the water of a seaplane in the planing condition. Reports and Memoranda No. 1493, Aeronautical Research Committee, London.

Privalov, E. I. \& Kirillovikh, V. N. 1996 Transport amphibious platforms: a new type of high-speed craft. In Workshop Proc. of Ekranoplans \& Very Fast Craft, Sydney, Australia, 5 December 1996, pp. 121-133. 
Roskam, J. 1989 Airplane Design Part I : Preliminary Sizing of Airplanes. Ottawa: Roskam Aviation and Engineering Corporation.

Rozhdestvensky, K. V. 1996 Ekranoplans - the GEM's of fast water transport. Trans. Inst. Marine Eng. 109, 47-74.

Rozhdestvensky, K. V. 2006 Wing-in-ground effect vehicles. Prog. Aerosp. Sci. 1, 211-283. (doi:10.1016/j.physletb.2003.10.071)

Savitksy, D. 1964 Hydrodynamic Design of Planing Hulls. J. Mar. Technol. 1, 71-95.

Savitsky, D., De Lorme, M. F. \& Datla, R. 2007 Inclusion of Whisker Spray Drag in Performance Prediction Method for High-Speed Planing Hulls. Mar. Technol. 44, 3556.

Shipps, P. R. 1976 Hybrid ram-wing/planning craft - Today's raceboats, tomorrow's outlook. In AIAA/SNAME Advanced Marine Vehicles Conf., Arlington, VA, 20 September 1976, pp.1-8.

Staufenbiel, R. W. \& Bao-Tzang, Y. 1977 Stability and control of ground effect aircraft in longitudinal motion. Report (translation), David W. Taylor Naval Ship Research and Development Center.

Troesch, A. W. 1992 On the Hydrodynamics of Vertically Oscillating Planing Hulls. J. Ship Res. 36, 317-331.

Troesch, A. W. \& Falzarano, J. W. 1993 Modern Nonlinear Dynamical Analysis of Vertical Plane Motion of Planing Hulls. J. Ship Res. 37, 189-199.

Ward, T. M., Goelzer, H. F. \& Cook, P. M. 1978 Design and performance of the ram wing planing craft - KUDU II. In AIAA/SNAME Advanced Marine Vehicles Conf., SanDiego, California, 17 April 1978, pp. 1-10

Zarnick, E. E. 1978 A Nonlinear Mathematical Model of Motions of a Planing Boat in Regular Waves. Report no. DTNSRDC 78-032, David W. Taylor Naval Ship Research and Development Center, Carderock, Md. 